\title{
The numerical modeling of gas liquid two-phase flow in Laval nozzle
}

\author{
Hongyue Wang \\ School of Hongyue Wang, North China Electric Power University, Baoding 071000, China \\ 1658263043@qq.com
}

Keywords: Expansion and dehydration, Laval nozzle, CFD, FLUENT.

\begin{abstract}
In recent years, many country are vigorously developing natural gas mining technology, the supersonic expansion dehydration is a new natural gas dehydration methods, which has many advantage. Based on this background, this paper aimed to analyze the flow field in supersonic expansion dehydration of Laval nozzle and to study its effects on the whole process of dehydration. This paper firstly design for the Laval nozzle, after finding the reasonable parameters, using CFD method, the FLUENT software to carry on the numerical simulation, the analysis and the results in different angles.
\end{abstract}

\section{Introduction}

In recent years, our country is to develop the gas drilling technology, and gas coming up from underground or maritime transport natural gas with a large number of moisture, is not conducive for the transport and use. At present, aimed at removing the water in natural gas, many scholars have done a lot of research, and natural gas dehydration methods are varied, adiabatic throttling, turbine expansion, low temperature cooling, etc. But these methods have large energy consumption, has the disadvantages such as pollution. In this background the supersonic expansion encapsulationdehydration can well solve these problems. It is mainly composed of Laval nozzle, cyclone, and diffuser. Contains a large amount of water vapor in natural gas after a Laval nozzle, due to its own pressure, temperature sharply reduce, lead to condensation water, again through the cyclone to water droplets on the wall, achieve the purpose of dehydration. The dehydration device, small size, low consumption, high efficiency, no pollution, so the scholars pay a lot of attention.

\section{The design of the Laval nozzle}

\subsection{Throat design}

Now commonly used BWRS equation to calculate the critical parameters of inlet temperature, pressure, throat size can be determined in the end. According to one dimensional is entropic flow.

When not considering the influence of the boundary layer, under the critical flow condition, the equation of the gas flow inside the nozzle is:

$G=\rho_{t} V_{t} A_{c r}$

Where $G t$ represent the gas flow, $\rho_{t}$ represent the gas density, $V t$ is the gas flow rate, and Acr is the throat area.

According to (2-1) we can obtain:

$A_{c r}=\frac{G}{\rho_{t} V_{t}}$

And because:

$A_{c r}=\frac{\pi d_{0}}{4}$

If it is an ideal gas, according to the thermodynamics, the flow and area formula is:

$G=\sqrt{k\left(\frac{2}{k+1}\right)^{\frac{k+1}{k-1}}} \frac{P_{0}}{\sqrt{R_{m} T_{0}}} A_{c r}$ 


$$
A_{c r}=G / \sqrt{k\left(\frac{2}{k+1}\right)^{\frac{k+1}{k-1}}} \frac{P_{0}}{\sqrt{R_{m} T_{0}}}
$$

Where $\mathrm{k}$ is specific heat ratio, Po is the entrance to the stagnation pressure, $R m$ is gas constant.

In the actual gas, we should first find out gas critical parameters and then calculate the throat size. It is due to the air in the nozzle is one dimensional isentropic flow and there is no external work output so:

$$
\begin{aligned}
& S_{t}-S_{0}=0 \\
& \frac{V_{t}^{2}}{2}=h_{0}-h_{1}
\end{aligned}
$$

When the flow is critical:

$$
a=V_{t}
$$

Where So represents the stagnation state entropy of gas imports, $S t$ is The throat of the entropy, $h o$ is Gas state stagnation enthalpy of imports, $h t$ is The throat of the enthalpy, $V t$ is throat velocity.

According to the speed of sound formula:

$$
a=\sqrt{k_{v} Z_{t} R_{m} T_{t}}
$$

Mouth and throat area depends on the gas flow, when the gas is saturated vapor, throat diameter is:

$$
d_{c r}=1.57 \sqrt{\frac{G}{p}} m m
$$

Where $P$ represents absolute pressure $\mathrm{kgf} / \mathrm{mm} 2$.

\section{2 contraction section design}

There are many method of contraction curve design; the commonly used methods are as follows: quintal curve, Batchelor-Shaw, Vito Eric hinske curve and Double cubic curve. Different design methods apply to different scope, here are mainly introduces the following four methods.

Quintal curve

Equation:

$$
\frac{D-D_{c r}}{D_{1}-D_{c r}}=1-10\left(\frac{X}{l_{1}}\right)^{3}+15\left(\frac{X}{l_{1}}\right)^{4}-6\left(\frac{X}{l_{1}}\right)^{5}
$$

\subsection{Expansion period of design}

Expansion curve shape design method mainly has: darfur and the method, the design method of characteristic line method, the law and tapered tube method, area ratio formula.

furshy method

This method is characteristic of spray tube wall exterior curve analysis method can be calculated when designing. Practice has proved that using this method to design the nozzle flow of quality can meet the design requirements. There are three sections of curves according to this method of design the supersonic expansion curve.

Specific steps are as follows:

1) First of all, Design Mach number according to the throat diameter and divergent section of export Me, Cross sectional area and divergent section of exports Ae:

$$
\frac{A_{E}}{A_{c r}}=\frac{M_{c r}}{M_{E} \times\left\{\left[1+\frac{(k+1) M_{E}{ }^{2}}{2}\right] /\left[1+\frac{(k+1) M_{c r}^{2}}{2}\right]\right\}^{\frac{(k+1) M_{c r}^{2}}{2(k-1)}}}
$$

Where Acr represents Nozzle throat cross-sectional area, Ae is Nozzle expansion period of export cross-sectional area, $\mathrm{K}$ is specific heats of gases, $\mathrm{M}$ is divergent section of nozzle exit Mach number, Mcr is Nozzle throat critical Mach number.

2) According to the following formula and divergent section of the exit Mach number Me, we can obtain the dilation Angle here and the specific value $\tau_{E}$ of exit Radius and critical radius $r_{E}$ : 


$$
\begin{aligned}
\psi & =\frac{\sqrt{\frac{k+1}{k-1}} \times \arctan \sqrt{\frac{k-1}{\left(M^{2}-1\right)(k+1)}}-\arctan \sqrt{M^{2}-1}}{2} \\
\tau_{p}^{2} & =\frac{\left\{\left[2+(k-1) M_{P}^{2}\right] /(k+1)\right\}^{\frac{k+1}{2(k-1)}}}{M_{P}}
\end{aligned}
$$

3) Use the relational expression $\omega=1 / 2, \psi_{E}=\theta_{A}=\psi_{A}$, then use (2-13) and (2-14) to calculate $M_{A}$ and $\tau_{A} \cdot \theta_{P}=\psi_{E}-\psi_{P}$ is a point on Mach line

4) choose mach Mp range from $M_{A}$ to $\mathrm{Me}$, use (2-13) to calculate $\psi_{P}$, then use the following equations to calculate coordinates $\mathrm{x}$ of $\mathrm{AB}$ line.

$$
\begin{aligned}
& x=\frac{D_{E}}{4 \sin (\omega / 2)} \frac{\tau_{P}}{\tau_{E}} \frac{1+\left[\cos \theta_{P} \sqrt{M_{P}^{2}-1}-\sin \theta_{P}\right] \times F\left(\theta_{P}\right)}{\sin \theta_{P} \times \sqrt{M_{P}^{2}-1}+\cos \theta_{P}}-X_{0} \\
& y=\left\{D_{E} /[4 \sin (\omega / 2)]\right\} \times \tau_{P} F\left(\theta_{P}\right) / \tau_{E}
\end{aligned}
$$

In the equations:

$$
\begin{aligned}
& F\left(\theta_{P}\right)=\sqrt{\sin ^{2} \theta_{P}+2\left(\cos \theta_{P}-\cos \varpi\right)\left(\sqrt{M_{P}^{2}-1}+\cos \theta_{P}\right)} \\
& x_{0}=\frac{D_{E}}{2 \tau_{E}} \times\left\{\operatorname{ctg} \varpi-\left[\frac{\tau_{A} \cos (\varpi / 2)-1}{2 \cos (\varpi / 2)[\sin (\varpi / 2)+\cos (\varpi / 2)]}\right]\right\}
\end{aligned}
$$

5) We can calculate $\mathrm{R}$ and $\lambda$ according to the following equation, where $\mathrm{R}$ is excessive arc radius of curvature throat, $\lambda$ is the length of the straightway.

$$
R=\lambda=\frac{D_{E}}{4 \tau_{E} \sin (\varpi / 2)} \times \frac{\tau_{A} \cos (\varpi / 2)-1}{2 \cos (\varpi / 2)+\sin (\varpi / 2)}
$$

\section{CFD numerical modeling}

\subsection{Geometric model}

As the result of the cyclone separator is axisymmetric structures, using two-dimensional numerical calculation model. Through the above calculation, the geometric model size is as shown in figure 3-1, here set up the model as the basic model.

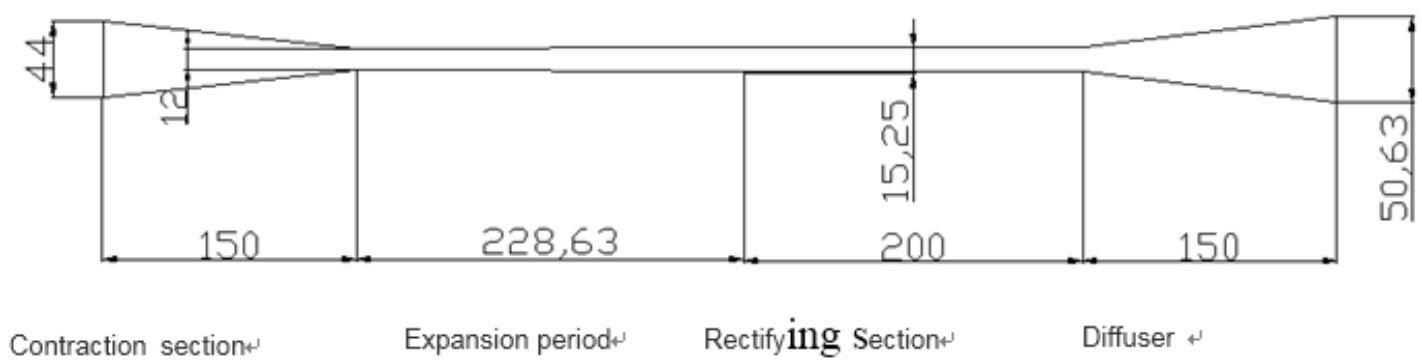

Figure 1

With two-dimensional model of spiral flow blade not well express, therefore in the model we ignore it. Figure 4-3 geometric model is obtained by simplifying and cyclone separator, this paper mainly studies nozzle and back-end rectifier tube and the inside of the diffuser pressure, temperature, and the change of the velocity distribution.

\subsection{The results of numerical calculation and analysis}

After the preparation of separator is simulated, and the velocity vector diagram, the pressure distribution, and so on, respectively, as shown in figure 3-3, figure 3-4. Can clearly see from the picture, in the temperature and pressure in the nozzle interval gradually decline, rapidly increases, in the throat location Mach number is 1 . The air flow inside the nozzle throat section has reached 
supersonic state, and has kept increasing speed. Due to the nozzle airflow in adiabatic expansion, so that the temperature and pressure to reduce gradually, thus forming the low temperature low pressure. Nozzle outlet temperature drop to $-57^{\circ} \mathrm{C}$, pressure drop to 31.2 ATM, reach supersonic airflow speed obviously, highest can reach $411 \mathrm{~m} / \mathrm{s}$. Low temperature low pressure environment, create conditions for condensate water and heavy diameter appear light condensate water and heavy. When the temperature reaches the dew point temperature, the gas has been saturated state or oversaturated, droplets will occur. But the airflow pressure rising too fast in the rectifying section, temperature, flow velocity let start decreasing, and the velocity gradient is bigger, no longer for supersonic speed. What we need is in the rectifying section is relatively stable state. In the divergent section, pressure and temperature rise gradually. By the law of conservation of energy, gradually reduce the speed, the kinetic energy into pressure energy, increased the air pressure. This is bad for vapor liquid effectively separated, directly reduce the separation efficiency.

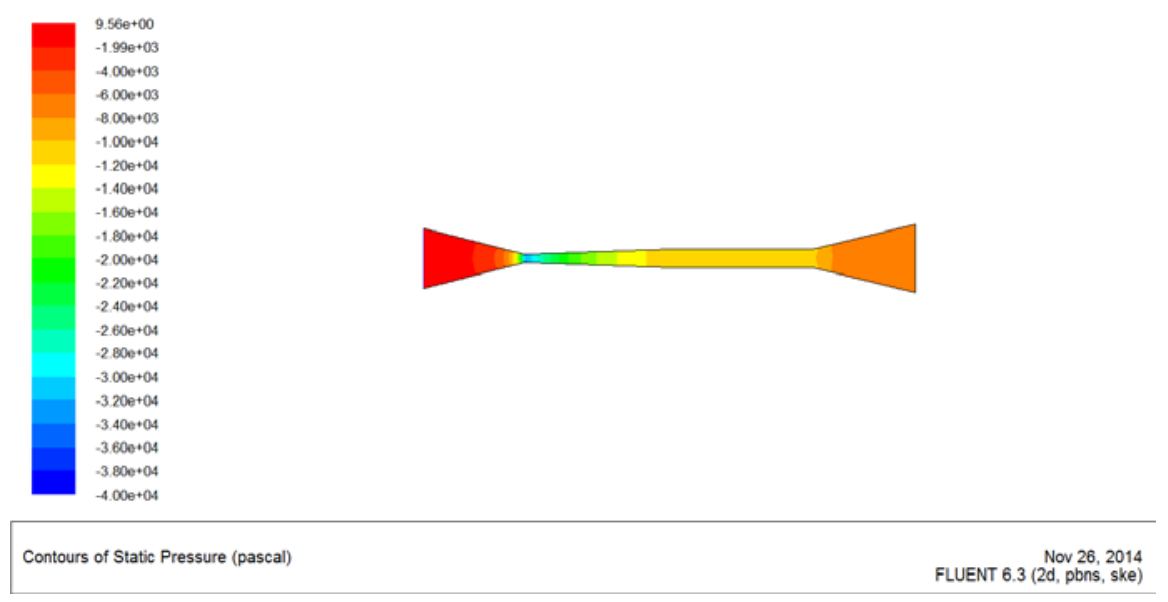

Figure 2

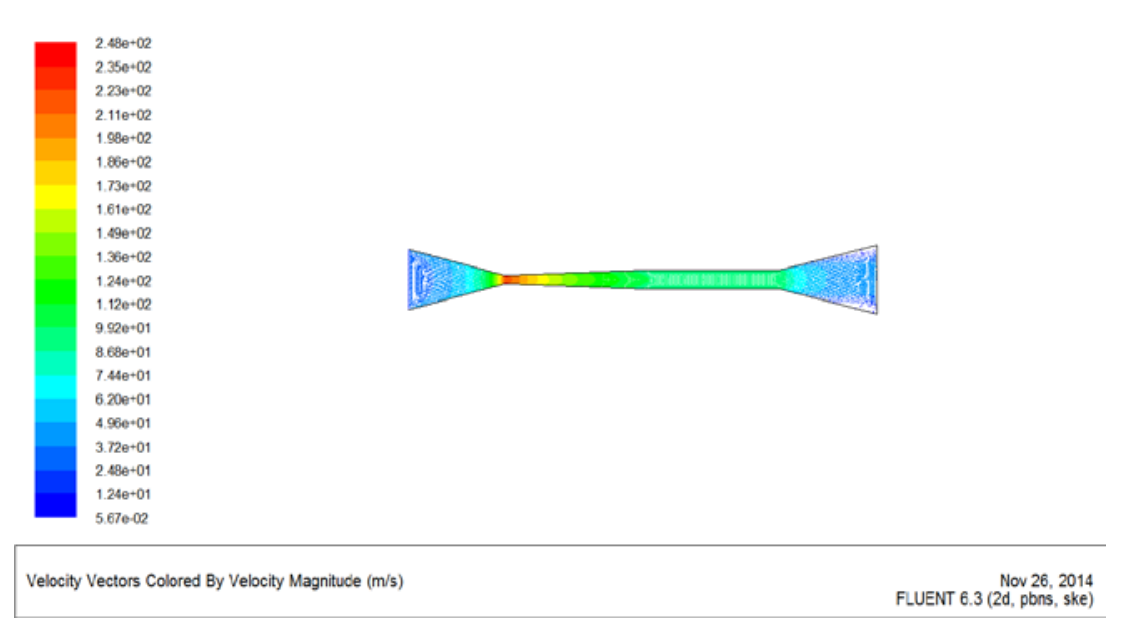

Figure 3

\section{Conclusions}

Supersonic expansion in natural gas dehydration process was studied in detail in this paper, an important part of the Laval nozzle is the influence of various parameters on the dehydration effect, first of all, by calculating the Laval nozzle size design, then introduces the method of CFD and using FLUENT software to carry on the numerical simulation, analyzes the throat size, diffuser outlet diameter changes, separation hydro cyclone length and diameter expansion period of export parameters on the effect of the supersonic separator and get a reasonable size. For natural gas dehydration unit provides a good idea, has a wider significance. 


\section{Reference}

[1]. Jiang Wen ming, The supersonic separation tube two-dimensional numerical simulation and analysis of the internal flow, Journal of Engineering Thermo physics, December, 2008.

[2]. Yang Fan, Gas-liquid two phase flow cyclone separator studies at a high speed, master's thesis, Xi'an Shi you University. 20th, May, 2011.

[3]. Liu Feng, Natural gas supersonic flow condensation process in the separator, refrigeration technique, the 11th edition. 10

[4]. Hu Shi jun, Supersonic jet vortex tube gas separation performance study, Dalian University of Technology Press, Jun, 2011.

[5]. Liang Bing zhi, Supersonic gas liquid separator for natural gas dehydration process improvement is analyzed, $\mathrm{Xi}$ 'an petroleum university press, 2014.

[6]. Chuan shao liu,Numerical simulation of gas-particle two phase flow in Aluminum-oxide ceramics powder spraying process ,henan polytechnic University.

[7]. Fred T.Okimoto, Marco Betting 2001 Laurance Reid Gas Conditioning Conferencr Norman ,Oklahoma 2001,2.

[8]. Bart Prast, Peter Schinkeshoek, Bart Lammers, Marco 2005 Betting Adances in Multiphase 6. 